\title{
Energy based model for temperature dependent behavior of ferromagnetic materials
}

\author{
Sanjay Sah and Jayasimha Atulasimha* \\ Department of Mechanical and Nuclear Engineering, Virginia Commonwealth University, Richmond, \\ Virginia 23284, USA. \\ * Address correspondence to: jatulasimha@vcu.edu
}

\begin{abstract}
An energy based model for temperature dependent anhysteretic magnetization curves of ferromagnetic materials is proposed and benchmarked against experimental data. This is based on the calculation of macroscopic magnetic properties by performing an energy weighted average over all possible orientations of the magnetization vector. Most prior approaches that employ this method are unable to independently account for the effect of both inhomogeneity and temperature in performing the averaging necessary to model experimental data. Here we propose a way to account for both effects simultaneously and benchmark the model against experimental data from $\sim 5 \mathrm{~K}$ to $\sim 300 \mathrm{~K}$ for two different materials in both annealed (fewer inhomogeneities) and deformed (more inhomogeneities) samples. This demonstrates that this framework is well suited to simulate temperature dependent magnetic behavior.

\section{Introduction}

There have been many magnetization models for ferromagnetic and magnetostrictive materials such as Preisach model [1-2], Weiss model [3], Stoner-Wolfforth model [4], Brown's analysis of thermal fluctuation in singe domain particles [5], homogenized energy model [6], Jiles-Atherton model [7-8], energy weighted models [9-13], Globus model [14] other nonlinear constitutive [15] and phase field approaches [16]. While models such as the Preisach model are purely mathematical and do not actually addresses the underlying physics, later models attempt to incorporate specific exchange coupling, shape anisotropy, magnetoelastic anisotropy magnetocrystalline anisotropy and Zeeman energies in describing the magnetization behavior of bulk samples. However, the saturation magnetization, magnetocrystalline

anisotropy and average magnetic domain volumes change with a change in temperature. This can present a challenge in modeling temperature dependent magnetization behavior of such materials accurately. In this paper we propose an energy based approach which can comprehensively model the magnetic behavior of ferromagnetic materials over a range of temperatures by correctly accounting for these temperature effects and benchmark this model against experimental data.

One potential application of the proposed model is to simulate the behavior of passive ferromagnetic shielding materials that are very important for the proper function of cryomodules of particle accelerators [17], sensitive probes and detectors used in satellites and spacecrafts [24], and any other instruments requiring isolation from external magnetic
\end{abstract}


fields. Furthermore, this modeling framework can also be extended to model magnetostriction and would hence be useful for the design of magnetostrictive actuators and sensors that are used over a wide range of temperatures [25-28]. Magnetostrictive actuators, operating over a wide range of temperatures (cryogenic to room temperature), have recently found some niche applications [25-28].

Many prior models approximately employ the following approach [6-14] or some variants thereof to model the magnetization behavior. The total energy density $\left(\mathrm{E}_{\mathrm{i}}\right)$ corresponding to the magnetization orientation along a crystallographic direction (" $i$ ") as shown in figure 1(a) is evaluated and the probability $\left(\mathrm{p}_{\mathrm{i}}\right)$ of this state being occupied is calculated as:

$$
p_{i}=\frac{e^{-\frac{E_{i} V}{k T}}}{\sum_{i} e^{-\frac{E_{i} V}{k T}}}
$$

Here $\mathrm{k}$ denotes the Boltzmann constant, $\mathrm{T}$ the temperature and $\mathrm{V}$ is the volume as discussed below.

However, there are two challenges in applying such models to modeling ferromagnetic behavior over a wide range of temperature, even if these are well below the Curie temperature $\left(\mathrm{T}_{\mathrm{c}}\right)$ : (i) the definition of "V" is nebulous at best for bulk samples and may approximately be assumed to correspond to the average size of a magnetic domain. Even this poses an issue as $\mathrm{V}$ may change as domains form, coalesce, etc. during the magnetization process. (ii) At low temperatures (say when $\mathrm{T} \sim$ few Kelvin) this model will only permit the minimum energy states to be occupied that will tend to simulate magnetization curves as shown in figure 1 (b), which do not model experimental magnetic behavior at low temperatures correctly.

Some models $[9,10,13]$ consider the effect on inhomogeneities (defects, grain boundaries, polycrystalline texture, etc.) on the possibility of occupation of non-minimum energy states. This is an important reason for magnetization cures not looking like figure 1 (b) at low temperatures. These models calculate the probability $\mathrm{p}_{\mathrm{i}}$ of occupation of state as:

$$
p_{i}=\frac{e^{-\frac{E_{i}}{\Omega_{o}}}}{\sum_{i} e^{-\frac{E_{i}}{\Omega_{o}}}}
$$

Here, they use an empirical term $\Omega_{\mathrm{o}}$ with no temperature dependence. Hence, both models described by (1) and (2) do not have a framework to model magnetization over a range of temperatures while comprehensively accounting for the effects of magnetic field, magnetocrystalline anisotropy, stress anisotropy, defects, etc. Therefore, we propose to model both effects simultaneously by defining $\Omega$ as follows:

$$
\Omega=\Omega_{\mathrm{o}}+\Omega_{1}\left(\frac{\mathrm{T}}{\mathrm{T}_{\mathrm{c}}}\right)
$$

Hence, there is an explicit dependence of the occupation of non-minimum states on defects and inhomogeneities (through $\Omega_{\mathrm{o}}$ ) as well as temperature (through $\Omega_{1}\left(\mathrm{~T} / \mathrm{T}_{\mathrm{c}}\right)$ ). It is evident that as $\Omega_{\mathrm{o}}$ increases or $\Omega_{1}\left(\mathrm{~T} / \mathrm{T}_{\mathrm{c}}\right)$ increases, the high energy states are penalized less (larger denominator) and hence have high probability of being occupied. On the contrary, low $\Omega_{\mathrm{o}}$ (less inhomogeneity) and low $\Omega_{1} \quad\left(\mathrm{~T} / \mathrm{T}_{\mathrm{c}}\right)$ (low temperatures) would penalize the occupation of high energy states more severely.

\section{Model}

The model is implemented by considering the total energy density for the magnetization orienting in a direction $\left(\alpha_{1}, \alpha_{2}, \alpha_{3}\right)$ in a cubical anisotropy material due to an applied magnetic field $(\mathrm{H})$ with direction cosine $\left(\beta_{1}, \beta_{2}, \beta_{3}\right)$, with respect to crystallographic axes. For this paper, we only consider the magnetocrystalline energy 
and Zeeman energy contributions (we note stress anisotropy and other contributions may be added where appropriate):

$$
\begin{gathered}
E=E_{\text {magnetocrystalline }}+E_{\text {magnetic }} \\
=K_{1}\left(\alpha_{1}^{2} \alpha_{2}^{2}+\alpha_{2}^{2} \alpha_{3}^{2}+\alpha_{3}^{2} \alpha_{1}^{2}\right)+K_{2}\left(\alpha_{1}^{2} \alpha_{2}^{2} \alpha_{3}^{2}\right) \\
-\mu_{0} M_{s} H\left(\alpha_{1} \beta_{1}+\alpha_{2} \beta_{2}\right. \\
\left.\quad+\alpha_{3} \beta_{3}\right)
\end{gathered}
$$

Where, $\mathrm{K}_{1}$ is the fourth order cubical anisotropy constant, $\mathrm{K}_{2}$ is the second order cubical anisotropy constant, and $\mathrm{M}_{\mathrm{s}}$ is the saturation magnetization. The shape anisotropy is not included as the experimental data is corrected for the effects of demagnetization (i.e. $\mathrm{H}_{\text {Effective }}$ $=\mathrm{H}_{\text {External }}-\mathrm{H}_{\text {Demagnetization }}$ is used in the experimental data and was estimated in the manner described in detail in Ref. [18]).

The total magnetization in the $\mathrm{z}$ - direction is then given as $[9,10]$ :

$$
\begin{array}{r}
<M_{Z}>=\sum M_{S} \cos \theta P_{i} \\
\mathrm{M}_{\mathrm{z}}=\frac{\left.\iint_{(\theta, \phi)}\left(\mathrm{M}_{s}(\mathrm{~T}) \cos \theta\right)(\mathrm{d} \theta \mathrm{d} \phi)|\sin \theta|\right) \mathrm{e}^{-\frac{\mathrm{E}}{\Omega}}}{\left.\iint_{(\theta, \phi)}(\mathrm{d} \theta \mathrm{d} \phi)|\sin \theta|\right) \mathrm{e}^{-\frac{\mathrm{E}}{\Omega}}}
\end{array}
$$

Here $M_{s}$ is the saturation magnetization, $\theta$ is the polar angle and $\phi$ is the azimuthal angle shown in figure 1(a). The integral is evaluated using the appropriate function for double integral in MATLAB.

Likewise, we could model the magnetostrictive behavior by including the magnetoelastic term in equation 4 and calculating the magnetostriction in the manner of Ref 9. However, this is not the focus of this paper where we discuss a framework to model a general class of ferromagnetic materials.

The magnetization model (equations 3, 4 and 5) only applies to temperatures well below the Curie temperature of the material which is $\sim 400^{\circ} \mathrm{C}$ [23]. The saturation magnetization $\left(\mathrm{M}_{\mathrm{s}}\right)$ and magnetocrystalline anisotropy $\left(\mathrm{K}_{1}\right)$ both are

(a)

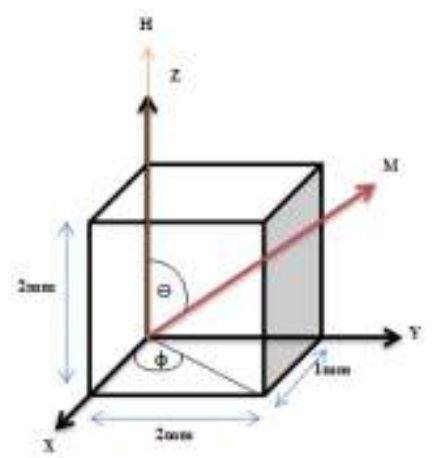

(b)

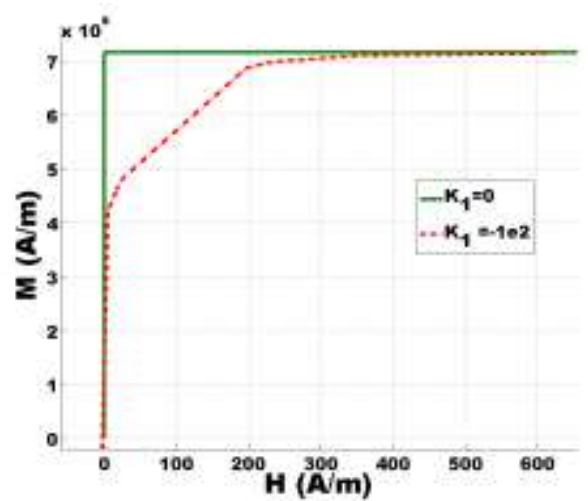

Figure 1. (a) Schematic of sample (b) $\mathrm{M}-\mathrm{H}$ curve at $5 \mathrm{~K}$ of annealed amumetal sample (at zero and non-zero magneto crystalline anisotropy; with $\Omega_{0}=0$ in both cases).

temperature dependent. Temperature dependence of $M_{s}$ is described as follows [8]:

$$
\frac{M_{S}(T)}{M_{S}\left(T_{o}\right)}=\left[\frac{T_{c}-T}{T_{c}}\right]^{a}
$$

Here, $M_{s}\left(T_{o}\right)$ is magnetization at $0 \mathrm{~K}$, Tc is the Curie temperature and $\alpha$ is material dependent critical exponent.

The temperature dependence of $\mathrm{K}_{1}$ is described as follows [22]:

$$
K_{1}=K_{1}\left(T_{o}\right) * \exp \left(-\beta T^{2}\right)
$$


Here $\mathrm{K}_{1}\left(\mathrm{~T}_{\mathrm{o}}\right)$ is $\mathrm{K}_{1}$ at $0 \mathrm{~K}$ and $\beta$ is material dependent empirical coefficient. Estimation of both $\alpha$ and $\beta$ is described in Appendix A.

\section{Discussion and analysis}

The magnetic characterization in Ref 18 was carried out using Quantum Design VersaLab and a SQUID magnetometer. We obtained experimental data [18] for two Ni-Cr-Fe alloys (Amumetal and $\mathrm{A} 4 \mathrm{~K}$ ) at different temperatures $(5 \mathrm{~K}$ to $300 \mathrm{~K}$. The samples were deformed plastically with the application of bending stress comprising of both tension and compression. The deformed samples were then restored to their original shape before performing magnetic characterization. We note that the "deformed" samples are not characterized under the application of any external stress. The plastic deformation/dislocations induced by the deformation process lead to a magnetic anisotropy in the manner of Ref [19] irrespective of the compressive/tensile nature of stress during the deformation process.

For the annealed sample, we assume we have very low or vanishing cubic anisotropies in the materials [20]. But the cubical anisotropies are induced in the deformed samples due to permanent deformation were accounted for with the $\mathrm{K}_{1}$ term [19].

The following procedure is applied to estimate the model parameters to simulate the behavior of amumetal and A4K bulk samples. These are NiCr-Fe alloys typically used for magnetic shielding in particle accelerators. The saturation magnetization $\left(\mathrm{M}_{\mathrm{s}}\right)$ was obtained from the experimental data for different temperatures. We note the experimental characterization does not show saturation here as the range of magnetic field is small, saturation magnetization $\left(\mathrm{M}_{\mathrm{s}}\right)$ is obtained from the experimental characteristics in Ref 18. $\mathrm{K}_{1}(\mathrm{~T})$ for the annealed samples was assumed to be zero [19]. $\mathrm{K}_{2}$ for all samples at all temperatures was assumed to be zero. For all annealed samples, $\Omega=\Omega_{\mathrm{o}}+\Omega_{1}\left(\mathrm{~T} / \mathrm{T}_{\mathrm{c}}\right)$ was chosen to give the best fit across all temperatures. For the deformed samples, $\Omega=\Omega_{\mathrm{o}}+\Omega_{1}\left(\mathrm{~T} / \mathrm{T}_{\mathrm{c}}\right)$ was chosen to give the initial slope of the curve and $\mathrm{K}_{1}(\mathrm{~T})$ was chosen to get the overall best fit. The best fit implies that the parameters are obtained from least square regression curve fitting with less than 5\% normalized root mean square error between the model and the experimental data in each case (each temperature) for figures 2 and 3. The error is experimental data due to uncertainty in the measurement of $\mathrm{H}$ and $\mathrm{M}$ is well within $5 \%$ as discussed in Ref 18.

The model parameters selected for amumetal and $\mathrm{A} 4 \mathrm{~K}$ are summarized in table 1.

(a)

(b)
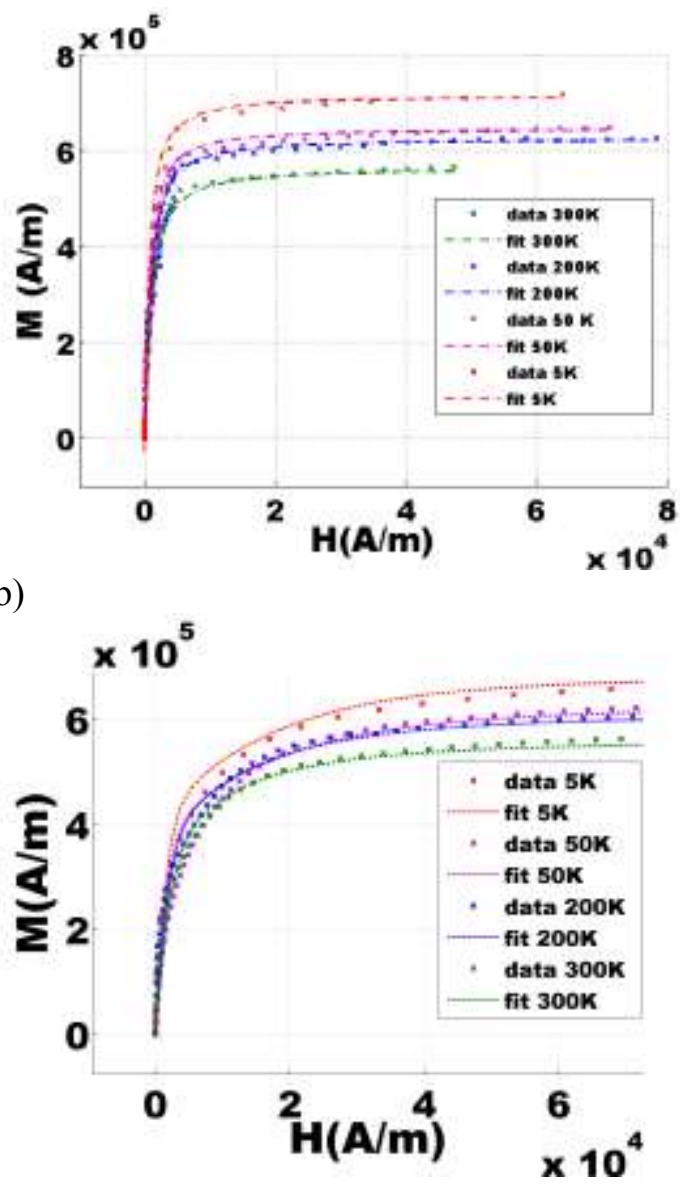
Figure 2. Comparison between simulated and experimental M-H curves for amumetal samples at different temperatures (a) Annealed amumetal sample with $\Omega=418+(0.21 \times \mathrm{T})(\mathrm{b})$ Deformed amumetal sample with $\Omega=910+(0.21 \times \mathrm{T})$ and empirically chosen $\mathrm{K}_{1}$.

Table 1. Modeling parameters $\Omega$ and $\mathrm{K}_{1}$

\begin{tabular}{|c|c|c|c|c|}
\hline & \multicolumn{2}{|c|}{ Undeformed } & \multicolumn{2}{|c|}{ Deformed } \\
\hline Amumetal & $K_{1}\left(J / m^{3}\right)$ & $\begin{array}{c}\Omega\left(J / \mathrm{m}^{3}\right) 418 \\
+(0.21 \times \mathrm{T})\end{array}$ & $\left|\mathbf{K}_{1}\right|\left(\mathbf{J} / \mathbf{m}^{3}\right)$ & $\begin{array}{c}\Omega\left(\mathrm{J} / \mathrm{m}^{3}\right) 910 \\
+(0.21 \times \mathrm{T})\end{array}$ \\
\hline $\begin{array}{l}5 \mathrm{~K} \\
50 \mathrm{~K} \\
200 \mathrm{~K} \\
300 \mathrm{~K}\end{array}$ & $\begin{array}{l}0 \\
0 \\
0 \\
0\end{array}$ & $\begin{array}{l}419 \\
429 \\
460 \\
481\end{array}$ & $\begin{array}{l}1.59 \times 10^{4} \\
1.51 \times 10^{4} \\
1.09 \times 10^{4} \\
7.06 \times 10^{3}\end{array}$ & $\begin{array}{l}911 \\
921 \\
952 \\
973\end{array}$ \\
\hline A4K & & $\begin{array}{c}410 \\
+(0.24 \times T)\end{array}$ & & $\begin{array}{c}880 \\
+(0.24 \times T)\end{array}$ \\
\hline $\begin{array}{l}5 \mathrm{~K} \\
50 \mathrm{~K} \\
200 \mathrm{~K} \\
300 \mathrm{~K}\end{array}$ & $\begin{array}{l}0 \\
0 \\
0 \\
0\end{array}$ & $\begin{array}{l}411 \\
422 \\
458 \\
482\end{array}$ & $\begin{array}{l}8.39 \times 10^{3} \\
8.18 \times 10^{3} \\
5.90 \times 10^{3} \\
3.03 \times 10^{3}\end{array}$ & $\begin{array}{l}881 \\
892 \\
928 \\
952\end{array}$ \\
\hline
\end{tabular}

(a)

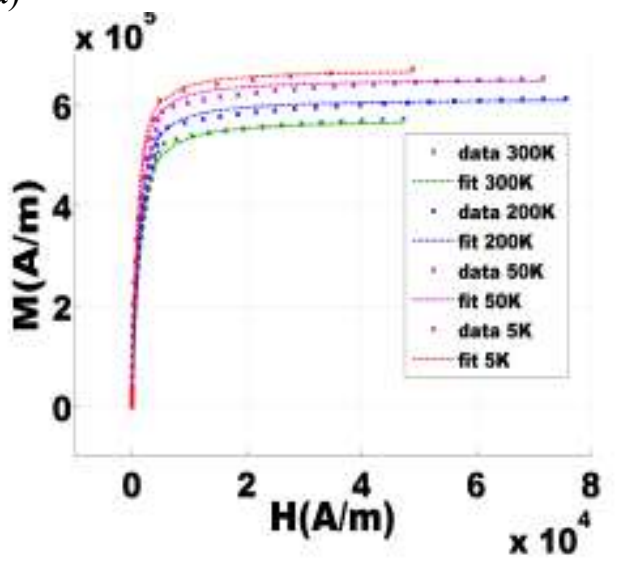

(b)

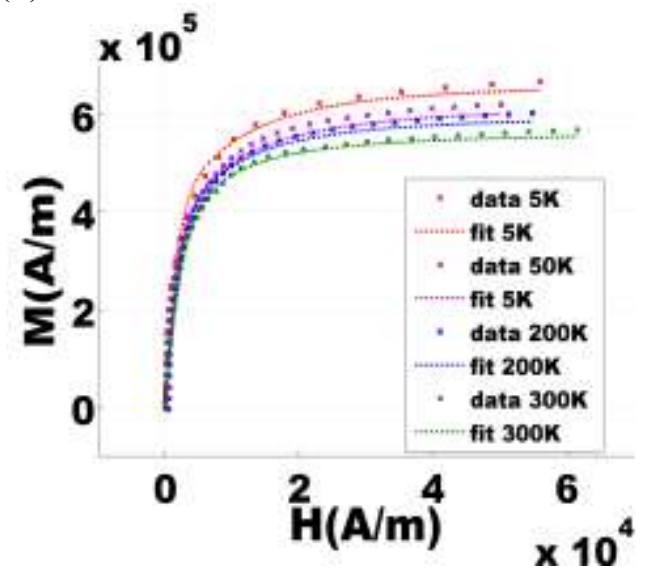

Figure 3. Comparison between simulated and experimental $\mathrm{M}-\mathrm{H}$ curves for $\mathrm{A} 4 \mathrm{~K}$ samples at different temperatures: (a) Annealed A4K sample with $\Omega=410+(0.24 \times \mathrm{T})$ (b) Deformed A4K sample with $\Omega=$ $880+(0.24 \times \mathrm{T})$ and empirically chosen $\mathrm{K}_{1}$. 
Now, we compare the magnetization models using the conventional approach and the new proposed approach for $\Omega$. For the new approach we propose we have:

$$
\Omega_{\mathrm{i}}=\Omega_{\mathrm{o}}+\Omega_{1}\left(\frac{\mathrm{T}}{\mathrm{T}_{\mathrm{c}}}\right)
$$

For the old approach we have:

$$
\Omega_{\mathrm{ii}}=\frac{\mathrm{KT}}{\mathrm{V}}=\text { Constant } *(\mathrm{~T})
$$

For the A4K annealed sample, we compare the results from both approaches and examine the differences. The results for the model using $\Omega_{\mathrm{i}}$ are already presented in figure 3 . The value of $\Omega_{\mathrm{ii}}$, is computed at $5 \mathrm{~K}$ to find the constant term in equation 9 that would best fit the $5 \mathrm{~K}$ data. Then the value of $\Omega_{\mathrm{ii}}$ is computed at $300 \mathrm{~K}$ using the constant term and equation 9. The results from both the approaches are plotted against experimental data as shown in figure 4 for comparison. While the simulated results from the new approach (equation 8) give us excellent fit with the experimental results at $5 \mathrm{~K}$ and $300 \mathrm{~K}$, the conventional method's simulated results completely fail to fit the experimental results at $300 \mathrm{~K}$. Likewise, if we had tried to fit the $300 \mathrm{~K}$ data with the conventional approach, the simulated results for the $5 \mathrm{~K}$ data would have failed to fit the experimental data.

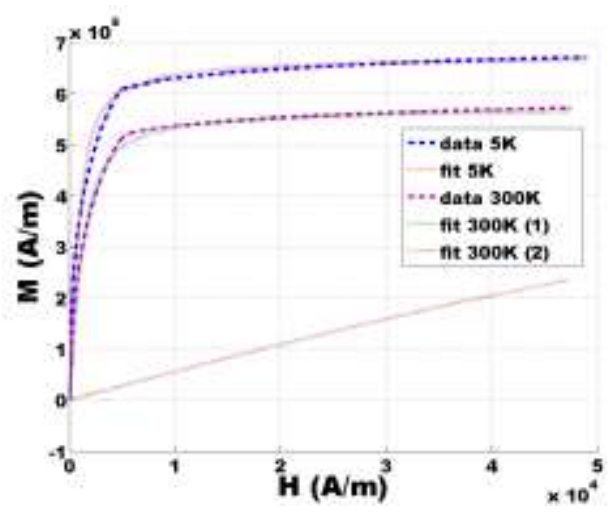

Figure 4. Comparison of conventional approach and new approach for the simulation of $\mathrm{M}-\mathrm{H}$ curves of A4K annealed sample at $5 \mathrm{~K}$ and $300 \mathrm{~K}$. This gives the distinct differences between the use of $\Omega_{\mathrm{i}}$ (see fit $300 \mathrm{~K}(1)$ ) and $\Omega_{\mathrm{ii}}$ (see fit $300 \mathrm{~K}(2))$.

\section{Conclusion}

In conclusion, we presented a modified energy based temperature dependent model that could simulate the magnetization of ferromagnetic materials over a range of temperatures by simultaneously incorporating the effect of inhomogeneity and temperature. As expected, $\Omega_{0}$ is smaller for annealed samples than deformed samples as the former have less inhomogeneity than the latter, where defects are induced during the deformation process. $\Omega_{\mathrm{T}}=\Omega_{1}\left(\mathrm{~T} / \mathrm{T}_{\mathrm{c}}\right)$ is independent of the processing (i.e. same for annealed or deformed samples) of a given material. This implies that $\Omega_{\mathrm{T}}$ purely models the effect of temperature, independent of the $\Omega_{\mathrm{o}}$ term that only incorporates the effect of inhomogeneity. The $K_{1}$ (cubic anisotropy) is induced by deformation and its value decreases with increasing temperature as higher temperatures can quench the anisotropy induced by the deformation [21]. In summary, we propose an approach for modeling temperature dependent magnetic behavior of ferromagnetic materials, which is well suited to simulate temperature dependent experimental magnetic behavior.

\section{Acknowledgement}

We acknowledge collaboration between Virginia Commonwealth University (VCU) and Jefferson Lab (U.S. DOE Contract No. DE-AC0506OR23177) that partly supports Sanjay Sah.

\section{References}

[1] Preisach E 1935 Ueber die magnetische Nachwirkung. Zeitschrift fuer Physik, 94:277-302. 
[2] Bergqvist A and Engdahl G 1991 IEEE Transactions on Magnetics, 27, 47964798.

[3] Weiss P 1907, J. Phys. 6,667.

[4] Stoner E C and Wohlfarth E P 1948, Philosophical Transactions of the Royal Society A: Physical, Mathematical and Engineering Sciences 240 (826): 599642.

[5] Brown W F 1963 Jr., Phys. Rev. 130, 1677.

[6] Smith R C , Dapino M J , Braun T R, and Mortensen A P 2006 IEEE

Transactions on Magnetics, VOL. 42, No. 7.

[7] Jiles D C and Atherton D L 1986, "Theory of ferromagnetic hysteresis," JMMM, vol. 61, pp. 48-60 .

[8] Raghunathan A, Melikhov Y, Snyder J E, and Jiles D C 2010, IEEE Transactions on Magnetics, Vol. 46, No. 6.

[9] Armstrong W D 2000 Materials Science and Engineering A: Structural Materials: Properties, Microstructure and Processing, 285, 13-17.

[10] Atulasimha J, Flatau A B, and Cullen J R 2008 J. Appl. Phys. 103, 014901.

[11] Chakraverty S, and M. Bandyopadhyay M 2007 J. Phys.: Condens Matter 19216201.

[12] Bandyopadhyay M 2009 , J. Phys.: Condens. Matter 21236003.

[13] Evans P G and Dapino M J 2009, Journal of Applied Physics 105, 113901.

[14] Globus A 1975 , Physical Society, Conference on Soft Magnetic Material 2, p. 233.

[15] Carman G P, and Mitrovic M 1995, Journal of Intelligent Material Systems and Structures, V. 6, No. 5, pp. 673-683.
[16] Ni Y and Khachaturyan A G 2007, J. Appl. Phys. 102, 113506.

[17] Cheng G , Daly E F , and Hicks W R 2008, "C100 Cryomodule Magnetic Shielding Finite Element Analysis", JLAB-TN-08-015.

[18] Sah S, Myneni G and Atulasimha J 2015, IEEE Transactions on Magnetics, Volume: 52 Issue: 2.

[19] Bhattacharyya A, Pollack S R, and Maddin R, 1966 Journal of Applied Physics 37, 4443.

[20] Couderchon G, Portesell J L, Bertotti G 1989 IEE Trans. On Magnetics, Vol. 25, No. 5.

[21] Rodbell D S 1962, Journal of Applied Physics 33, 1126.

[22] Honda K, Masumoto H, and Shirakawa Y 1935 "Science Reports of." Tohoku Imperial Uni.

[23] Private communication and material data sheet from AMUNEAL Manufacturing Corp.

[24] Kamiya K, Warner B A, Dipirro M J, and Numazawa T 2003 Physica B: Condensed Matter Volumes 329-333, Part 2, Pages 1627-1628

[25] Teter J P, Horner G C and Bromberg L 2000 J. Appl. Phys. 87, 6313

[26] Hathaway K B and Clark A E 1993 "Magnetostrictive materials." MRS Bulletin 18.04: 34-41.

[27] Ghodsi M, Ueno T, Teshima H, Hirano H, Higuchi T, and Summers E 2007 Sensors and Actuators A 135 787-791

[28] Weisensel G N, McMasters O D, and Chave R G 1998, Smart Structures and Materials :Industrial and Commercial Applications of Smart Structures Technologies. Vol. 3326.

\section{APPENDIX A}


The variation of $M_{s}$ with temperature, $M_{s}(T)$, was determined by fitting the experimental data to equations 6 as shown in figure A1.

The variation of $\mathrm{K}_{1}$ with temperature, $\mathrm{K}_{1}(\mathrm{~T})$, was determined by fitting estimates of $\mathrm{K}_{1}$ at different temperatures to equations 7 as shown in figure A2. It must be noted the estimates of $\mathrm{K}_{1}$ at each temperature was obtained to best model the experimental $\mathrm{M}-\mathrm{H}$ curve at that temperature and hence these estimates are based on experimental data.

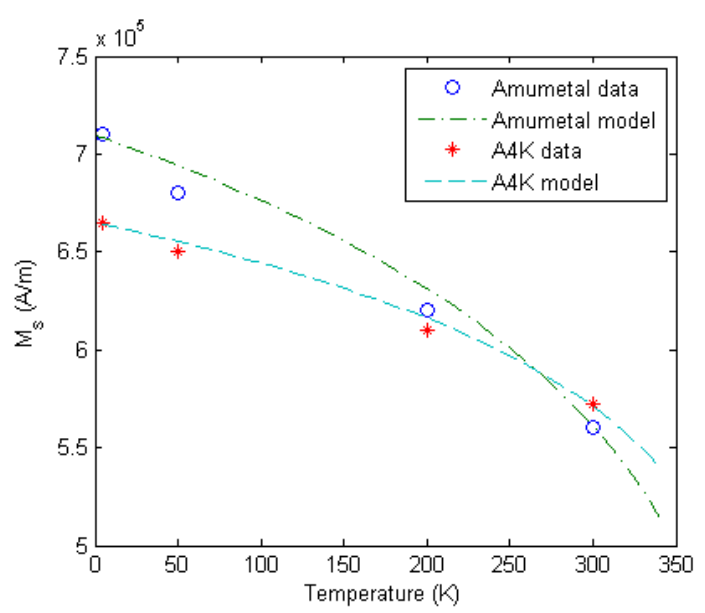

Figure A1. Variation of $\mathrm{M}_{\mathrm{s}}$ of Amumetal and $\mathrm{A} 4 \mathrm{~K}$ as a function of temperature (with $\alpha=0.17$ for Amumetal and $\alpha=0.11$ for $\mathrm{A} 4 \mathrm{~K}$ ).

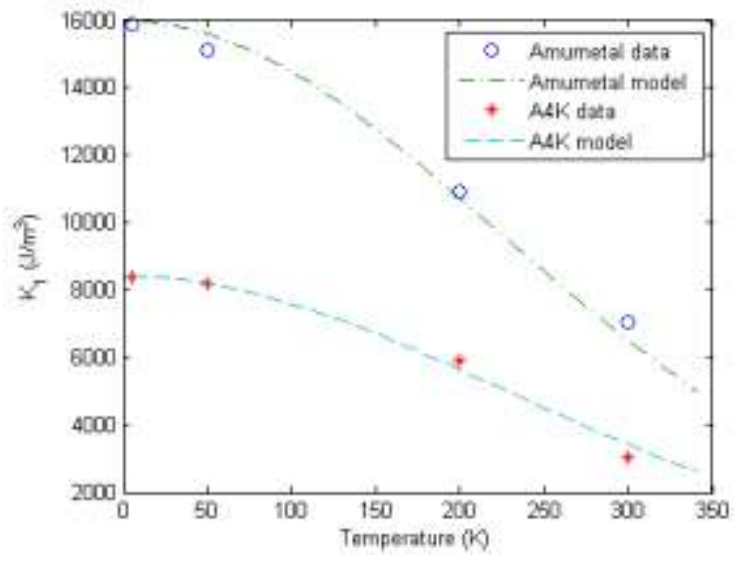

Figure A2. Variation of $\mathrm{K}_{1}$ of Amumetal and $\mathrm{A} 4 \mathrm{~K}$ as a function of temperature with $\mathrm{a}=-$ $1 \times 10^{-5}$. 